\title{
Estudo randômico da correção cirúrgica do prolapso uterino através de tela sintética de polipropileno tipo I comparando histerectomia versus preservação uterina
}

\section{A randomized comparison of two vaginal procedures for the treatment of uterine prolapse using polypropylene mesh: histeropexy versus histerectomy}

Silvia Carramão"; Antonio Pedro Flores Auge²; Aparecida Maria Pacetta3; Eliana Duarte ${ }^{5}$; Paulo Ayrosa ${ }^{4}$; Nucélio lMl lemos ${ }^{6}$; TSUTOMU AOKI ${ }^{7}$

\section{RE S U M O}

\begin{abstract}
Objetivo: Comparar os resultados anatômicos pós-operatórios de pacientes portadoras de prolapso uterino tratadas utilizando tela de polipropileno para correção dos defeitos do assoalho pélvico, comparando histerectomia vaginal com a preservação do útero. Método: Estudo randomizado com 31 mulheres portadoras de prolapso uterino estádio III ou IV (POP-Q) divididas em dois grupos: Grupo HV- 15 mulheres submetidas à histerectomia vaginal e reconstrução da anatomia do assoalho pélvico com tela de polipropileno tipo I (Nazca R-Promedon) e Grupo HP- 16 mulheres mulheres submetidas à reconstrução da anatomia do assoalho pélvico com tela de polipropileno tipo I (Nazca R-Promedon) preservando o útero. Raça, urgência miccional, constipação intestinal, dor sacral, sangramento e tempo de operação foram os parâmetros analisados. Resultados: O tempo de seguimento médio foi de nove meses. Não se observou diferença entre os grupos nas complicações funcionais. O tempo cirúrgico foi 120 minutos para grupo HV versus 58.9 minutos para grupo HP ( $p<0.001$ ) e o volume de perda sanguínea intraoperatória foi $120 \mathrm{~mL}$ no grupo HV versus 20 $\mathrm{mL}$ para grupo HP $(\mathrm{p}<0.001 *)$. A taxa de sucesso objetivo foi $86.67 \%$ para grupo HV e $75 \%$ para grupo HP $(p=0,667)$. A taxa de erosão de tela foi $20 \%(3 / 15)$ de extrusão no grupo HV versus $18,75 \%(3 / 16)$ no grupo HP $(p=1,000)$. Conclusão: A correção cirúrgica do assoalho pélvico com telas nas portadoras de prolapso uterino apresentaram similaridade quer sendo ela feita com histeropexia quer com histerectomia. Contudo, o tempo cirúrgico e o volume da perda sanguínea foram significantemente maiores no grupo com histerectromia (HV). Operações vaginais com telas são procedimentos efetivos para a correção do prolapso.
\end{abstract}

Descritores: Histerectomia. Prolapso uterino. Soalho pélvico/surgery. Polipropilenos/uso terapêutico. Telas cirúrgicas.

\section{INTRODUÇÃO}

O prolapso de órgãos pélvicos é definido como o deslocamento permanente, parcial ou total, de qualquer segmento vaginal ou órgão pélvico da sua localização habitual, abrangendo a procidência das paredes vaginais ou do útero'. Estima-se ${ }^{2}$ que $50 \%$ das mulheres multíparas percam o suporte pélvico adequado desenvolvendo algum grau de o prolapso. Para a melhor compreensão da fisiopatologia das distopias genitais é necessário o conhecimento da teoria de DeLancey ${ }^{3}$ que divide as estruturas que sustentam a vagina em três níveis (I, II e III), correspondentes a três diferentes áreas ou grupos de suporte ${ }^{4}$. De acordo com o compartimento e o nível acometido, obtém-se um tipo de prolapso genital. Com a inversão da parede vaginal, as mulheres experimentam grande desconforto para caminhar ou sentar, obstipação intestinal, alterações miccionais, ulcerações vaginais, infecções recorrentes do trato urinário e dificuldade ao coito ${ }^{5}$.

A correção cirúrgica do prolapso apical pode ser realizada por via vaginal ou abdominal ${ }^{6}$. Isto decorre porque na atualidade ainda não há consenso sobre a melhor técnica para a correção do prolapso uterino ${ }^{7}$. Historicamente o reparo do prolapso uterino por via vaginal tem sido realizado através da histerectomia associada à fixação da cúpula vaginal aos ligamentos útero-sacros (McCall) ou pela fixação ao ligamento sacro-espinhal. Entretanto, os índices de falha são descritos entre 4 e 33\% $\%^{8,9}$ necessitando de novos procedimentos cirúrgicos para correção.

A conduta convencional na correção do prolapso uterino é a histerectomia vaginal, com a finalidade de diminuir o risco de recorrência do prolapso. A fáscia

\footnotetext{
Trabalho realizado no Departamento de Obstetrícia e Ginecologia da Faculdade de Ciências Médicas da Santa Casa de São Paulo. 1. Mestre em Tocoginecologia. Faculdade de Ciências Médicas da Santa Casa de São Paulo, SP, BR; 2. Professor adjunto, Chefe da Clínica de Uroginecologia, BR; 3. Professora assistente do Hospital das Clínicas da Faculdade de Medicina da USP, SP, BR; 4. Professor assistente do Departamento de Obstetrícia e Ginecologia, Santa Casa de Misericórdia de São Paulo, SP, BR; 5. Especialista em Tocoginecologia Pós graduanda pela Faculdade de Ciências Médicas da Santa Casa de São Paulo, SP, BR; 6. Doutor em Tocoginecologia da Faculdade de Ciências Médicas da Santa Casa de São Paulo, SP, BR; 7. Professor Adjunto da faculdade de Ciências Médicas da Santa Casa de São Paulo, $\mathrm{SP}, \mathrm{BR}$.
} 
endopélvica faz parte do aparelho de suspensão do assoalho pélvico e oferece suporte ao mesmo por meio de ligamentos e fáscias que são constituídos por fibras de colágeno interligadas com elastina, células musculares lisas e estruturas vasculares ${ }^{10}$. Os ligamentos têm, portanto, função simultânea de condutor neurovascular e estrutura de suporte ${ }^{11}$. Entretanto, ao realizar-se a histerectomia vaginal para correção do prolapso uterino, existe o risco de recidiva do prolapso vaginal devido à fragilidade dos tecidos lesados previamente, além do risco de lesão durante a dissecção cirúrgica ${ }^{12}$. Vários procedimentos cirúrgicos foram propostos para a correção do prolapso genital; no entretanto, apesar do crescente desenvolvimento das técnicas cirúrgicas e do conhecimento da anatomia pélvica, os resultados não são completamente satisfatórios. As taxas de recidivas do prolapso de órgãos pélvicos após correção cirúrgica variam de $22 \%$ a $92 \%{ }^{13,14}$.

O uso de telas na cirurgia reconstrutora pélvica se baseia na premissa de que as distopias são consequentes ao enfraquecimento dos tecidos naturais. Porém, o uso de material sintético no organismo também induz respostas inflamatórias e cicatriciais que podem ser indesejadas tais como: infecção, formação de seromas, erosão e formação de fístulas. Após a formação da cicatriz, pode ocorrer retração da tela em até $20 \%$. Tal reação pode ser desejada ou inconveniente, quando causa efeitos compressivos ou obstrutivos. Portanto, tal fenômeno deve sempre ser lembrado e a tela implantada sem tensão ${ }^{13}$.

A utilização de tela monofilamentar e macroporosa (tipo I) é a mais apropriada, porém na literatura são descritas taxas de extrusão dessa tela entre 6 e $25 \%{ }^{15}$.

Em estudo prospectivo ${ }^{16}$ avaliaram-se 168 muIheres portadoras de prolapso uterino, realizando histerectomia abdominal e sacrocolpofixação com tela em um grupo, sacrohisteropexia com tela em um segundo e histerectomia abdominal com sacrocolpofixação sem tela em um terceiro. Concluiu-se que a preservação do útero não aumentou a recidiva do prolapso genital. A recidiva do prolapso genital foi 6,2 vezes maior após histerectomia abdominal e sacrocolpofixação sem tela, demonstrando que o uso de telas é necessário na correção dos prolapsos genitais.

Em outro estudo prospectivo, 75 mulheres portadoras de prolapso uterino avançado foram divididas em dois grupos, comparando histerectomia versus preservação do útero, reconstruindo a anatomia do assoalho pélvico com tela "sling" intravaginal posterior. Após 29 meses de seguimento, houve um caso de recidiva do prolapso uterino no grupo com preservação uterina, mas $89,9 \%$ das mulheres estavam satisfeitas com a operação. A extrusão da tela ocorreu em 13,6\% no grupo histerectomizado versus $11,4 \%$ no grupo de preservação uterina. Os autores concluíram que a correção do prolapso uterino com tela sintética é eficaz e segura ${ }^{17}$.

Pelo exposto, justifica-se a necessidade do desenvolvimento de técnicas cirúrgicas menos invasivas, que preservem a anatomia e as funções do assoalho pélvico feminino, o que estimulou o desenvolvimento deste estudo, que objetiva comparar os resultados anatômicos pósoperatórios de pacientes portadoras de prolapso uterino tratadas por meio de tela de polipropileno para correção do defeito apical, comparando histerectomia vaginal (HV) com a histeropexia(HP).

\section{MÉTODO}

Trata-se de estudo randômico, multicêntrico, prospectivo, envolvendo 31 mulheres com prolapso apical estádio III ou IV POP-Q/ICS ${ }^{18}$, atendidas ambulatorialmente no período de junho de 2006 a dezembro de 2007. Todas concordaram em participar após leitura e assinatura do termo de consentimento livre e esclarecido, aprovado pelo Comitê de Ética em Pesquisa em Seres Humanos da Irmandade da Santa Casa de Misericórdia de São Paulo e pela Comissão Científica do Departamento de Obstetrícia e Ginecologia da mesma entidade e da Faculdade de Medicina $A B C$.

A divisão dos grupos foi realizada com tábua de randomização por um terceiro elemento não envolvido no estudo, que colocou os resultados obtidos em envelopes lacrados, os quais foram abertos no momento da internação da paciente em dois grupos: Grupo HV - 15 pacientes submetidas à histerectomia vaginal e correção do defeito apical com a utilização de tela sintética de polipropileno monofilamentar tipo I (Nazca R-Promedon), tendo os braços da tela fixados ao ligamento sacro-espinhal; Grupo HP - 16 pacientes submetidas à correção cirúrgica do defeito apical com a utilização de tela sintética de polipropileno monofilamentar tipo I (Nazca R ${ }^{\mathrm{R}}$ - Promedon), tendo os braços da tela fixados ao ligamento sacro-espinhal, preservando o útero.

Os critérios de inclusão foram: portadoras de prolapso uterino estádio III ou IV, com idade entre 48 e 80 anos. Os de exclusão foram: portadoras de neoplasia cervical ou uterina, portadoras de tumores ovarianos, doenças crônicas que impossibilitem o tratamento cirúrgico, apresentando ASA 3, prolapso retal e antecedente de correção de prolapso com uso de implantes, infecções urinárias ou vaginais; distúrbios de coagulação sanguínea; sistema imunológico comprometido ou qualquer outra condição que pudesse comprometer a cura; insuficiência renal e/ou obstrução do trato urinário superior; irradiação prévia.

As pacientes foram submetidas na consulta inicial e nos retornos de 1, 3, 6 e 12 meses à história clínica, exame físico geral e ginecológico. A estática pélvica foi avaliada segundo as recomendações da International Continence Society (ICS), segundo o sistema POP-QErrol Indicador nåo definido. e pelo Índice de Quantificação de Prolapso (POP-Q-I) Absoluto e Relativo ${ }^{19}$.

Para o rastreamento da incontinência urinária de esforço oculta todas as pacientes foram submetidas a estudo urodinâmico, com redução do prolapso, utilizando-se as 
recomendações da ICS. As pacientes que apresentavam diagnóstico pré-operatório de IUE, foram submetidas à correção cirúrgica por "sling" de tela de polipropileno tipo I sub-uretral transobturatório (Safyre TOे).

Foram avaliados o tempo cirúrgico (minutos), medido com cronômetro sendo utilizado para comparação somente o tempo da realização da operação e o volume de sangramento intra-operatório $(\mathrm{mL})$, o qual foi medido através de aspirador cirúrgico de plástico descartável, acoplado a coletor graduado a cada $20 \mathrm{~mL}$.

Todas as pacientes operadas foram submetidas ao uso de antibiótico profilático, empregando-se duas doses endovenosas de cefazolina sódica $1,0 \mathrm{~g}$ sendo a primeira no início da operação e a segunda quatro horas após a primeira, e à uma dose de metronidazol $500 \mathrm{mg}$ endovenoso no início do procedimento cirúrgico.

Os dados coletados foram alocados em planilha de cálculo do programa Excel do pacote Office da Microsoft@ (Excel: 2003). Os cálculos foram realizados por meio do programa SigmaStat da Jandel Corporation $\circledast$ (SigmaStat?;1995). Para a comparação entre os grupos quanto à idade, IMC, antecedentes obstétricos, POP-Q-I pré-operatório, POP-Q-I pós-operatório, tempo de operação (minutos) e volume de sangramento $(\mathrm{mL})$, foi utilizado o teste Mann-Whitney (Siegel, 1988). Foram feitas duas tabelas de freqüência dos procedimentos cirúrgicos prévios e concomitantes de acordo com os grupos (risco á $<5 \%)$.

\section{RESULTADOS}

Trinta e duas pacientes foram envolvidas no estudo e 31 avaliadas com seguimento médio de nove meses (grupo HV =9,2 meses; grupo HP $=7,5$ meses). Não houve diferença estatisticamente significante entre os grupos quanto ao tempo de seguimento. Uma paciente de 77 anos pertencente ao grupo HV, foi excluída do estudo por falecimento, decorrente de causas naturais (infarto do miocárdio) três semanas após o procedimento. Deste modo foram avaliadas 15 pacientes no grupo HV e 16 no grupo HP.

Para a análise da variável raça, considerou-se a cor de pele branca ou não branca. Os dois grupos, após análise estatística foram considerados homogêneos quanto à ela. A cor de pele branca foi predominante nos dois grupos estudados sendo no grupo HV de $87,5 \%$ (14/16) de brancas e $12,5 \%$ (2/16) de não brancas e no HP 75\% (12/ 16) de brancas e $25 \%$ (4/16) de não brancas sem diferença estatística $(p=0,654)$.

Também foram homogêneos em relação às variáveis: idade, índice de massa corpórea (IMC), número de gestações, paridade, número de partos vaginais, número de cesáreas e abortos (Tabela 1). As pacientes eram multíparas, sendo que prevaleceu o número de partos vaginais em relação à cesárea, nos dois grupos.
Quanto às operações prévias para incontinência urinária de esforço (IUE) o grupo HV apresentou três pacientes, uma com operação de Kelly Kenedy e duas de Burch, enquanto que o grupo HP não teve nenhuma. Em relação às operações concomitantes para IUE foi utilizada a técnica de Saphyre $T^{\circledR}$ em quatro do grupo HGV e em duas do HP.

Entre as 31 pacientes, 25 referiram ausência da atividade sexual, seis a mantinham, sendo que após o procedimento cirúrgico apenas uma do grupo HV referiu

Tabela 1 - Comparação de idade, índice de massa corpórea, número de gestações, partos vaginais, cesáreas e abortos entre os grupos HV e HP.

\begin{tabular}{|c|c|c|c|c|}
\hline & & $\mathrm{HV}$ & HP & $\begin{array}{c}\text { Teste de } \\
\text { Mann-Withney }\end{array}$ \\
\hline Idade & Média & 65,5 & 67,1 & \\
\hline \multirow[t]{3}{*}{ Anos } & Mediana & 69,0 & 67,5 & $P=0,620$ \\
\hline & Máximo & 76,0 & 77 & \\
\hline & Mínimo & 51 & 53 & \\
\hline \multirow[t]{4}{*}{ IMC } & Média & 26,4 & 23,8 & \\
\hline & Mediana & 25,6 & 24,2 & $P=0,123$ \\
\hline & Máximo & 36 & 27 & \\
\hline & Mínimo & 19 & 18 & \\
\hline \multirow[t]{4}{*}{ Partos Vaginais } & Média & 3,9 & 3,6 & \\
\hline & Mediana & 3,0 & 3,0 & \\
\hline & Máximo & 9 & 10 & \\
\hline & Mínimo & 2 & 0 & \\
\hline \multirow[t]{4}{*}{ Cesáreas } & Média & 0,2 & 0,0 & \\
\hline & Mediana & 0,0 & 0,0 & $P=0,064$ \\
\hline & Máximo & 1 & 0 & \\
\hline & Mínimo & 0 & 0 & \\
\hline \multirow[t]{4}{*}{ Abortos } & Média & 0,8 & 0,3 & \\
\hline & Mediana & 0,0 & 0,0 & $P=0,340$ \\
\hline & Máximo & 4 & 1 & \\
\hline & Mínimo & 0 & 0 & \\
\hline$\underline{N}$ & & 15 & 16 & \\
\hline
\end{tabular}

HV: Histerectomia Vaginal HP: Histeropexia IMC: Índice de massa corpórea $N=$ Amostra.

Tabela 2 - Dados relevantes do pós-operatório imediato das pacientes.

\begin{tabular}{lccc}
\hline Dias de internação & 3 dias & 3 dias & $p=1,000$ \\
\hline Lesão retal & $0 \%$ & $0 \%$ & $p=1,000$ \\
Lesão vesical & $0 \%$ & $0 \%$ & $p=1,000$ \\
Retenção urinária & $0 \%$ & $0 \%$ & \\
Urgência miccional & $26,67 \%$ & $31,25 \%$ & $p=0,779$ \\
& $(4 / 15)$ & $(5 / 16)$ & \\
Obstipação intestinal & $13,33 \%$ & $0 \%$ & $p=0,226$ \\
& $(2 / 15)$ & $(0 / 15)$ & \\
Dor sacral com irradiação & & & \\
para face posterior de coxa & $26,67 \%$ & $12,5 \%$ & $p=0,394$ \\
& $(4 / 15)$ & $(2 / 16)$ & \\
& &
\end{tabular}

HV: Histerectomia Vaginal HP: Histeropexia. 
dispareunia. Nesta amostra não foi referido a "hispareunia" (dor peniana durante o ato sexual).

Dados relevantes da evolução do pós-operatório imediato, como tempo de internação, lesão vesical, lesão intestinal, dor, sintomas de urgência miccional, obstipação intestinal são demonstrados na tabela 2 .

Nove pacientes apresentaram sintomas de urgência miccional, não havendo diferença estatística entre os grupos quanto a este sintoma $(p=0,779)$. Todas foram medicadas com oxibutinina $5 \mathrm{mg}$ via oral de 12/12 horas e apresentaram melhora da sintomatologia. Duas interromperam o uso da medicação após um mês de uso e não houve recidiva dos sintomas. A obstipação intestinal, após a operação, foi referida por duas pacientes do grupo HV, sendo orientada dieta rica em fibras e quando necessário, o uso eventual de laxante.

Tabela 3 - Comparação volume de sangramento intra-operatório entre os grupos HV e HP.

\begin{tabular}{lccc}
\hline & \multicolumn{3}{c}{ Volume de Sangramento $(\mathrm{mL})$} \\
& HV & HP & Total \\
\hline Média & 126,9 & 48,8 & 87,8 \\
Desvio padrão & 104,9 & 56,6 & 91,9 \\
CV Pearson\% & 82,7 & 116,1 & 104,7 \\
Mediana & 120 & 20 & 55 \\
Máximo & 460 & 200 & 460 \\
Mínimo & 20 & 5 & 40 \\
\hline
\end{tabular}

HV: Histerectomia Vaginal HP: Histeropexia $\mathrm{mL}$ : milímetros Teste de Kolmogorov-Smirnov Uma amostra $D=1,040 \quad p=0,230$ Duas amostras $D=1,414 \quad p=0,037$ * Teste de Mann-Whitney $Z=2,995 p=0,003^{*}$.
Seis pacientes apresentaram dor em região sacral irradiando para coxa, também não havendo diferença estatisticamente significante entre os grupos. As pacientes foram medicadas com cetoprofeno 50mg via oral de 12/12 horas por sete dias e complexo B um comprimido ao dia por três meses e todas referiram melhora da dor, entre um e três meses após a operação.

O volume de sangramento foi significantemente maior no grupo HV que apresentou mediana igual a 120 $\mathrm{mL}$, enquanto o grupo HP teve mediana igual a $20 \mathrm{~mL}$, com $p=0,003$ * (Tabela 3).

Quando aplicou-se o teste de KolmogorovSmirnov, considerando o total de 32 casos como uma única amostra, houve resultado não significante, ou seja, todas as pacientes vieram de uma população de distribuição normal. No teste para as duas amostras, considerando os

Tabela 4 - Comparação do tempo cirúrgico entre os grupos HV e HP.

\begin{tabular}{lccc}
\hline & \multicolumn{3}{c}{ Tempo cirúrgico } \\
& HV & HP & Total \\
\hline Média & 115,6 & 67,5 & 91,6 \\
Desvio padrão & 38,5 & 23,9 & 39,9 \\
CV Pearson\% & 33,3 & 35,4 & 43,5 \\
Mediana & 120 & 60 & 90 \\
Máximo & 180 & 110 & 180 \\
Mínimo & 50 & 40 & 40 \\
\hline
\end{tabular}

HV: Histerectomia Vaginal HP: Histeropexia min: minutos Teste de Kolmogorov-Smirnov. Uma amostra $D=1,086 p=0,189$ Duas amostras $D=1768 \quad p=0,004^{*}$ Teste de Mann-Whitney $Z=3,367 \quad p=0,001^{*}$.

Tabela 5 - Comparação do POP-Q e POP-Q-I, pré-operatório e pós-operatório no tempo máximo de seguimento, no grupo HV.

\begin{tabular}{|c|c|c|c|c|c|c|}
\hline \multirow[b]{3}{*}{ Variável } & \multicolumn{4}{|c|}{ Grupo HV } & \multirow{2}{*}{\multicolumn{2}{|c|}{ Teste de Wilcoxon }} \\
\hline & \multicolumn{2}{|c|}{ Pré-operatório } & \multicolumn{2}{|c|}{ Máx. seg. } & & \\
\hline & Média & Mediana & Média & Mediana & Z & $P$ \\
\hline $\mathrm{Aa}$ & 3,000 & 3,000 & $-0,533$ & $-1,000$ & 3,193 * & 0,001 * \\
\hline $\mathrm{Ba}$ & 6,200 & 6,000 & $-0,533$ & $-2,000$ & $3,413 *$ & 0,001 * \\
\hline $\mathrm{R} \mathrm{Ba}$ & 0,788 & 0,762 & 0,267 & 0,100 & $3,238 *$ & 0,001 * \\
\hline C & 7,233 & 7,000 & $-4,167$ & $-4,000$ & $3,418^{*}$ & $0,001 *$ \\
\hline$D-C$ & 15,900 & 15,000 & 2,333 & 1,500 & 3,410 * & 0,001 * \\
\hline Max D-C & 16,633 & 16,000 & 12,867 & 13,000 & $2,906 *$ & 0,004 * \\
\hline $\mathrm{RC}$ & 0,961 & 0,929 & 0,185 & 0,125 & $3,408 *$ & 0,001 * \\
\hline Ap & 2,333 & 3,000 & $-2,533$ & $-3,000$ & 3,451 * & 0,001 * \\
\hline $\mathrm{Bp}$ & 5,233 & 5,000 & $-2,600$ & $-3,000$ & $3,415^{*}$ & 0,001 * \\
\hline R Bp & 0,706 & 0,727 & 0,041 & 0,000 & 3,412 * & 0,001 * \\
\hline $\mathrm{D}$ & 6,533 & 6,000 & $-4,300$ & $-5,000$ & 3,422 * & 0,001 * \\
\hline R D & 0,877 & 0,875 & 0,173 & 0,115 & 3,351 * & 0,001 * \\
\hline CVT & 8,667 & 8,000 & 6,500 & 7,000 & 3,051 * & 0,002 * \\
\hline $\mathrm{HG}$ & 4,600 & 4,000 & 3,367 & 3,000 & $2,689 *$ & 0,007 * \\
\hline$C P$ & 2,500 & 2,000 & 3,233 & 3,500 & 2,694 * & 0,007 * \\
\hline
\end{tabular}

HV: Histerectomia Vaginal Máx. Seg.: Máximo Seguimento.

CVT: Comprimento Vaginal Total HG: Hiato Genital CP: Corpo Perineal. 
Tabela 6 - Comparação do POP-Q e POP-Q-I pré-operatório e pós-operatório no tempo máximo de seguimento no grupo HP.

\begin{tabular}{|c|c|c|c|c|c|c|}
\hline \multirow[b]{3}{*}{ Variável } & \multicolumn{4}{|c|}{ Grupo HP } & \multirow{2}{*}{\multicolumn{2}{|c|}{ Teste de Wilcoxon }} \\
\hline & \multicolumn{2}{|c|}{ Pré-operatório } & \multicolumn{2}{|c|}{ Máx. seg. } & & \\
\hline & Média & Mediana & Média & Mediana & Z & $p$ \\
\hline $\mathrm{Aa}$ & 2,233 & 3,000 & 0,467 & 0,000 & $2,166^{*}$ & 0,030 \\
\hline $\mathrm{Ba}$ & 4,433 & 5,000 & 1,067 & 2,000 & $2,912 *$ & 0,004 \\
\hline $\mathrm{R} \mathrm{Ba}$ & 0,671 & 0,700 & 0,406 & 0,500 & 2,482 * & 0,013 \\
\hline$C$ & 6,300 & 6,000 & $-1,667$ & $-4,000$ & 3,467 * & 0,001 \\
\hline$D-C$ & 14,367 & 14,000 & 5,467 & 2,500 & $3,466^{*}$ & 0,001 \\
\hline Max D-C & 15,533 & 14,000 & 13,333 & 14,000 & 1,910 & 0,056 \\
\hline $\mathrm{RC}$ & 0,934 & 0,964 & 0,465 & 0,167 & $2,844^{*}$ & 0,004 \\
\hline Ap & 2,467 & 3,000 & $-2,933$ & $-3,000$ & 3,621 * & 0,000 \\
\hline$B p$ & 4,300 & 4,000 & $-2,467$ & $-3,000$ & 3,537 * & 0,000 \\
\hline $\mathrm{R} \mathrm{Bp}$ & 0,660 & 0,667 & 0,047 & 0,000 & $3,523 *$ & 0,000 \\
\hline$D$ & 5,900 & 6,000 & $-2,600$ & $-4,000$ & 3,521 * & 0,000 \\
\hline R D & 0,873 & 0,861 & 0,311 & 0,167 & 3,517 * & 0,000 \\
\hline CVT & 8,067 & 7,500 & 7,133 & 7,000 & $1,698 *$ & 0,090 \\
\hline HG & 4,633 & 4,500 & 3,600 & 3,500 & 2,567 * & 0,010 \\
\hline$C P$ & 2,333 & 2,000 & 3,467 & 3,500 & 3,083 * & 0,002 \\
\hline
\end{tabular}

HP: Histeropexia Máx. Seg.: Máximo Seguimento.

CVT: Comprimento Vaginal Total HG: Hiato Genital CP: Corpo Perineal.

grupos HV e HP, houve significância, ou seja, os dois grupos comportam-se de modo diferente.

O tempo cirúrgico foi significantemente maior no grupo HV com mediana igual a 120 min, enquanto o grupo HP igual a 60 min ( $p=0,001$ ) (Tabela 4).

Quando aplicou-se o teste de KolmogorovSmirnov, considerando o total de 32 casos como uma única amostra, obteve-se resultado não significante, ou seja, todas as pacientes vieram de uma população normal. No teste para as duas amostras, considerando os grupos $\mathrm{HV}$ e HP, houve significância, ou seja, os dois grupos comportam-se de modo diferente.

Ao analisar-se os resultados anatômicos classificados pelo POP-Q ou pelo POP-Q I, comparando os resultados pré-cirúrgicos com os após o tempo máximo de seguimento, observou-se melhora significativa do grau de prolapso nos dois grupos, (Tabelas 5 e 6).

Os resultados anatômicos obtidos, avaliados pelo POP-Q e/ou POP-Q I (Tabela 7), demonstraram que não houve diferença estatística entre os dois grupos.

Para melhor visualização do resultado anatômico após os procedimentos cirúrgicos, expõem-se os resultados da mediana dos principais pontos do POP-Q na figura 1.

Nos dois grupos estudados considerando a recidiva de prolapso de parede vaginal anterior (PPVA) quando o ponto Ba estivesse abaixo de -1, observou-se $54,83 \%$ de recidiva do PPVA (17/31) após tempo máximo de seguimento. A recidiva do prolapso de parede posterior (PPVP) considerando o ponto Bp abaixo de -1, ocorreu em 2/ $31(6,45 \%)$.

A recidiva do prolapso apical ocorreu em quatro pacientes do grupo HP (25\%) e em duas pacientes do grupo HV $(12,5 \%)$, todas foram submetidas a novo procedimento cirúrgico, para correção do prolapso apical. A taxa de sucesso para a correção do prolapso apical foi de $86.67 \%$ para o grupo HV e $75 \%$ para o grupo HP não havendo diferença estatística entre eles $(p=0,664)$.

A taxa de erosão/extrusão da tela foi de 20\% (3/ 15) no grupo HV versus $18,75 \%(3 / 16)$ no grupo HP (p $=1,000$ ). As pacientes acometidas foram tratadas com estrogenioterapia tópica, utilizando creme com estriol (Ovestrion ${ }^{\mathrm{R}}$ ) por via vaginal, diariamente por três meses. As três pacientes do grupo HV e uma paciente do grupo HP necessitaram ressecção de aproximadamente $1 \mathrm{~cm}$ de tela da mucosa vaginal posterior e sutura das bordas da mucosa vaginal, tendo boa evolução.

Uma paciente do grupo HP teve $0,5 \mathrm{~cm}$ de tela extruída para o interior do reto, associada à recidiva do prolapso uterino e foi submetida à cirurgia com ressecção da tela for via retal e histerectomia vaginal com fixação da cúpula pela técnica de McCall, evoluindo satisfatoriamente após o procedimento.

Uma paciente do grupo HP com recidiva do prolapso uterino associada à extrusão de tela na mucosa vaginal posterior próxima ao ápice, também foi submetida à retirada parcial da tela e histerectomia vaginal complementada com técnica de McCall. Na dissecção do septo retovaginal, ocorreu lesão de aproximadamente dois centímetros da parede retal, a qual foi corrigida com sutura em dois planos com pontos de poliglactina 3-0 e a paciente evoluiu sem outras intercorrências.

As outras quatro pacientes com recidiva do prolapso apical foram submetidas à histerectomia vaginal e fixação da cúpula pela técnica de McCall, mantendo-se a tela, evoluindo sem intercorrências. 
Tabela 7 - Comparação do POP-Q e POP-Q I, pré-operatório e pós-operatório no tempo máximo de seguimento, entre os grupos HV e HP.

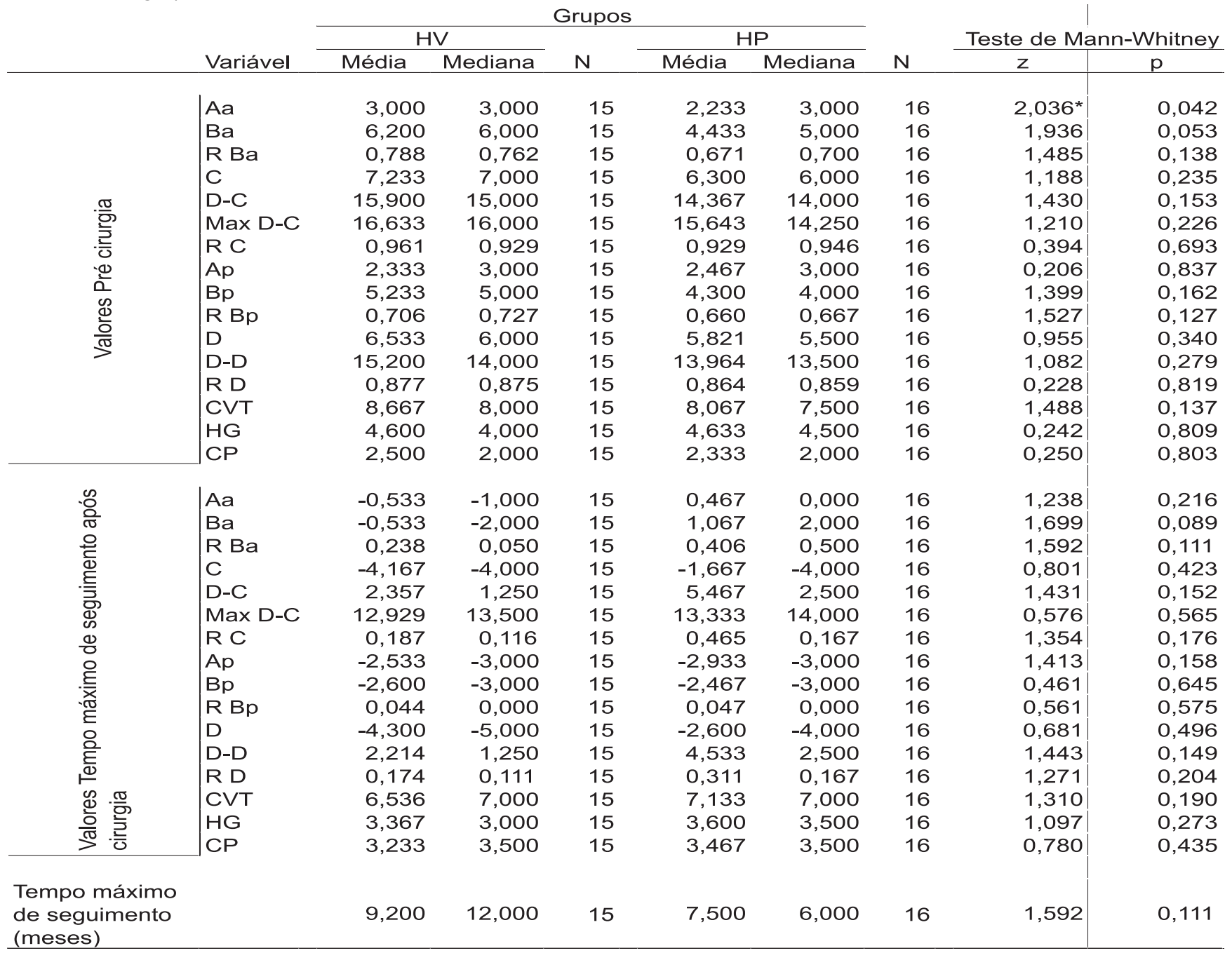

HV: Histerectomia Vaginal HP: Histeropexia N: Amostra.

\section{DISCUSSÃO}

O prolapso uterino acentuado é classicamente tratado por histerectomia vaginal, porém com a evolução do conhecimento anatômico, dos métodos propedêuticos

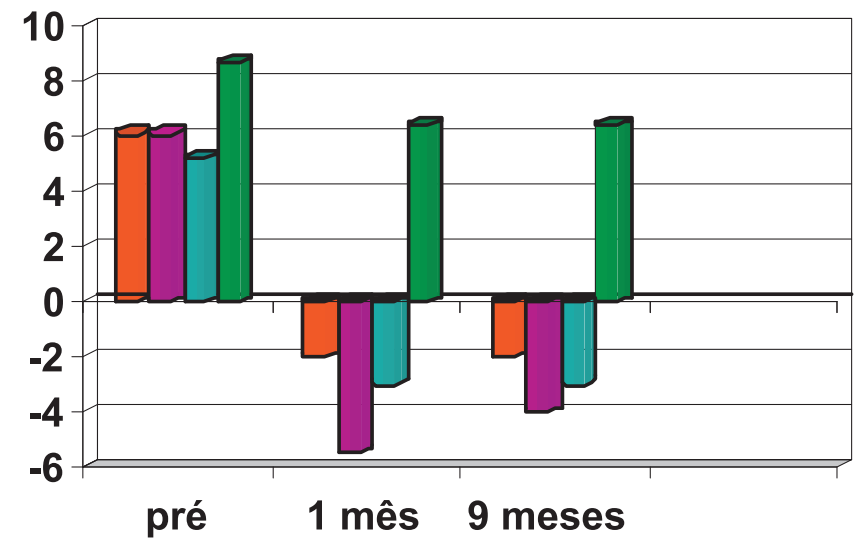

e das técnicas cirúrgicas, interroga-se o quanto a histerectomia é imprescindível para a correção do prolapso genital.

A desvantagem da histerectomia consiste na dissecção da fáscia endopélvica e secção dos ligamentos úte-
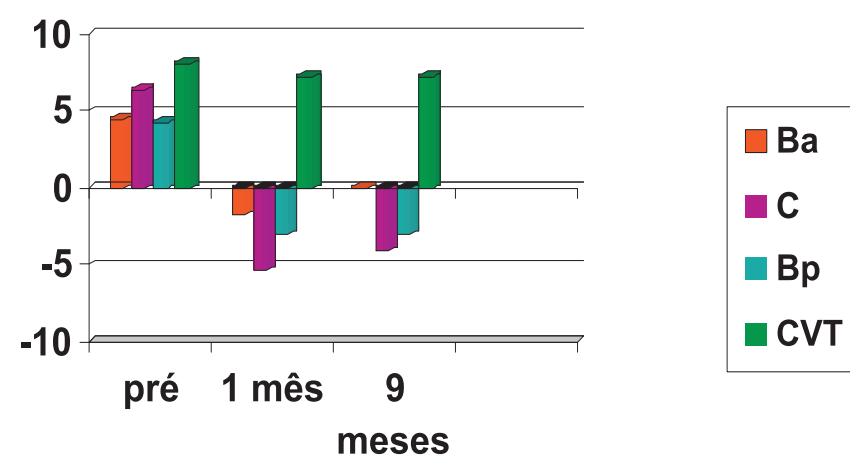

Figura 1 - Comparação do POP-Q (valores da mediana) pré-operatório e pós-operatório entre os grupos HV e HP. 
ro-sacros e cardinais, que resultará em processo de cicatrização com perda da qualidade do tecido fibromuscular como citado anteriormente por Nesbit ${ }^{20}$. Deste modo, ao realizar-se a histerectomia, está-se acentuando as lesões dos tecidos de sustentação e suspensão dos órgãos pélvicos, aumentando o risco de recidiva dos prolapsos. A operação de reconstrução do assoalho pélvico como tratamento do prolapso uterino pode conservar este órgão, tendo como benefício a preservação do anel fascial pericervical e a integridade do complexo ligamentar útero-sacro-cardinaiscardinais.

Weber ${ }^{21}$ citam em seu estudo que a recidiva do prolapso de parede vaginal é o calcanhar de Aquiles da reconstrução da anatomia do assoalho pélvico devido à alta recidiva deste prolapso, descrita em revisão de literatura entre 20 a $63 \%^{12,22}$. Neste estudo, apresentou-se $54,58 \%$ de recidiva do PPVA versus $6,45 \%$ de PPVP. A justificativa provável para esta ocorrência é ter-se corrigido os defeitos da parede vaginal anterior pela técnica sítio-específica, refletindo a má qualidade do tecido fibromuscular da fáscia pubocervical, frequentemente observada em pacientes portadoras de prolapsos genitais acentuados. Os resultados encontrados talvez sugiram a necessidade de correção do PPVA com tela sintética no mesmo procedimento, devendo ser foco de novo estudo.

$\mathrm{Na}$ literatura observa-se que os resultados anatômicos da correção do prolapso apical com telas sintéticas são promissores, porém este sucesso é obscurecido pela maior morbidade na utilização de telas, devido aos riscos de extrusão/erosão citados entre 6 e $25 \%{ }^{15,23}$. Em concordância, neste estudo observou-se $20 \%$ (3/15) de extrusão no grupo HV versus 18,75\%(3/ 16) no grupo HP. No total de seis extrusões em 31 pacientes submetidas à operação com tela de polipropileno, quatro apresentaram extrusões para a mucosa vaginal em pequena extensão, tendo resolução em procedimento ambulatorial evoluindo sem intercorrências, em concordância com o estudo de Deffieux et a ${ }^{24}$, que afirma que a conduta nas erosões/extrusões de telas sintéticas são simples e associadas com baixa morbidade. Porém a maior preocupação é com a extrusão da tela para outros órgãos, prejudicando a sua funcão e aumentando o risco de infecção e morbidade, como a extrusão da tela para o reto observada em uma paciente após 10 meses de seguimento, exigindo novo procedimento cirúrgico para sua ressecção e correção da recidiva do prolapso. Talvez o principal fator de risco para a extrusão de tela seja a fragilidade da mucosa vaginal decorrente do hipoestrogenismo.

As complicações após o uso de telas sintéticas necessitam de mais estudos prospectivos e randômicos para avaliação quanto à segurança e eficácia na correção dos defeitos apicais em longo prazo.
Embora algumas mulheres portadoras de prolapso uterino completo interrompam a atividade sexual devido à sua condição clínica, algumas manifestam o desejo de reiniciar a vida sexual após a correção do defeito. Atualmente as mulheres nascidas após a revolução sexual de 1960, estão atingindo a terceira idade, de modo que elas valorizam cada vez mais a função sexual, portanto, é importante o desenvolvimento de técnicas cirúrgicas que preservem a anatomia e função vaginal para esta geração de mulheres, mas que também sejam seguras para as pacientes mais idosas e portadoras de comorbidades.

A observação dos resultados no grupo HP utilizando-se de tela para a correção dos defeitos do assoalho pélvico quanto ao tempo cirúrgico (média igual a $60 \mathrm{mi}-$ nutos) e ao volume sanguíneo com média igual a 48,8 $\mathrm{mL}$, que foram significativamente menores, sugerem que este procedimento é rápido e seguro. DeLancey, $1997^{25}$, em operações de colpocleise observou tempo cirúrgico com média 101 minutos e o volume de perda sanguínea durante o procedimento com média de $206 \mathrm{~mL}$. Ao comparar-se os resultados dos dois estudos, pode-se observar que a correção do prolapso uterino com tela sintética possa ser um procedimento alternativo à colpocleise em pacientes idosas, sendo vantajoso por manter a anatomia e função vaginal.

Principalmente por não ter ocorrido diferença entre os dois grupos quanto à taxa de sucesso de correção anatômica e por ser a preservação do útero técnica de menor morbidade em termos de sangramento e tempo cirúrgico, a histerectomia não é necessária para a correção do prolapso uterino, desde que não haja outras doenças uterinas.

Ao revisar-se a literatura, observa-se que atualmente o cirurgião encontra diversas técnicas para a correção do prolapso uterino e alguns estudos sugerem a preservação do útero. A realização da histerectomia ou a preservação do útero para a correção do prolapso uterino, deve ser decisão conjunta de médico e paciente que, ao ser orientado, concorda com o procedimento. Tem-se que ter sempre em mente que a melhor técnica é a que associa segurança e melhor resultado cirúrgico com a maior satisfação à paciente.

Em conclusão, os resultados anatômicos pósoperatórios de pacientes portadoras de prolapso uterino tratadas por histerectomia vaginal ou preservação do útero seguidos da fixação da cúpula vaginal com tela posterior de polipropileno não apresentaram diferença estatisticamente significante entre eles. A correção cirúrgica do prolapso uterino acentuado, utilizando tela de polipropileno tipo I para reconstrução do assoalho pélvico, preservando ou não o útero, mostrou-se eficaz. 


\begin{abstract}
Objectives: To compare surgical morbidity and time, as well as anatomical outcomes between vaginal histerectomy and uterine preservation in the treatment of uterine prolapse using a mesh kit (Nazca $\mathbb{B})$. Methods: Randomized controled trial with 31 women with uterine prolapse POP-Q stage 3 or 4 pelvic organ prolapse who underwent vaginal surgery using tipe I polypropilene mesh (Nazca ()). They were randomized in two groups: group HV: hysterectomy and pelvic reconstruction floor with mesh ( $n=15)$; group HP: hysteropexy and pelvic reconstruction floor with mesh $(n=16)$. Race, miccional urgency, intestinal constipation, sacral pain were assessed as well as the amount of bleeding and time of operation. Results: Median follow-up was nine months on both groups. No difference was observed on complication rates and functional outcomes. Operation time was 120 minutes on group HV, versus 58.9 minutes on group HP $\left(X^{2}=17.613^{*}, p<0.001\right)$ and intraoperative blood loss was $120 \mathrm{~mL}$ on group HV versus $20 \mathrm{~mL}$ on group HP $\left(X^{2}=19.425^{*}\right.$; $p<0.001)$. There was no differences in relationship to anatomical cure rates. Objective success rate was $86.67 \%$ to group HV and $75 \%$ to group HP $(p=0,667)$ at nine months of follow-up. The anatomical results were similar between the two groups. Conclusion: The anatomic results between histeropexy and hysterectomy were similar. However, surgical time and blood loss were greater in group with histerectomies. The erosion rate were also similar. Vaginal surgery using mesh is an effective procedure for pelvic organ prolapse.
\end{abstract}

Key words: Hysterectomy. Uterine prolapse. Pelvic floor/surgery. Polypropylenes/ therapeutic use. Surgical mesh.

\section{REFERENCIAS}

1. Haddad JM, Pacetta AM, Neto MJ, Ribeiro RM. Prolapso genital e incontinência urinária de esforço. In: Oliveira HC, Lemgruber, editores. Tratado de Ginecologia FEBRASGO. Reimpressão. Rio de Janeiro: Revinter; 2001. p. 880-6.

2. Olsen AL, Smith VJ, Bergstrom JO, Colling JC, Clark AL. Epidemiology of surgically managed pelvic organ prolapse and urinary incontinence. Obstet Gynecol. 1997; 89(4): 501-6.

3. DeLancey JO. Anatomic aspects of vaginal eversion after hysterectomy. Am J Obstet Gynecol. 1992; 166(6 Pt 1):1717-24; discussion 1724-8

4. Auge AFP, Carramão SS. Prolapso de órgãos pélvicos. In: Piato S. Ginecologia diagnóstico e tratamento. São Paulo: Manole; 2008. p. 81-90.

5. Imparato E, Aspesi G, Rovetta E, Presti M. Surgical management and prevention of vaginal vault prolapse. Surg Gynecol Obstet. 1992;175(3): 233-7.

6. Maher C, Baessler K. Surgical management of posterior vaginal wall prolapse: an evidence-based literature review. Int Urogynecol J Pelvic Floor Dysfunct. 2006; 17(1): 84-8. Epub 2005 Apr 19.

7. Carey M, Slack M, Higgs P, Wynn-Williams M, Cornish A. Vaginal surgery for pelvic organ prolapse using mesh and a vaginal support device. BJOG. 2008; 115(3): 391-7.

8. Virtanen HS, Mäkinen Jl. Retrospective analysis of 711 patients operated on for pelvic relaxation in 1983-1989. Int J Gynaecol Obstet. 1993; 42(2): 109-15.

9. Benson JT, Lucente $\mathrm{V}$, McClellan E. Vaginal versus abdominal reconstructive surgery for the treatment of pelvic support defects: a prospective randomized study with long-term outcome evaluation Am J Obstet Gynecol. 1996; 175(6): 1418-21; discussion 1421-2.

10. DeLancey JO. Anatomy and biomechanics of genital prolapse. Clin Obstet Gynecol. 1993; 36(4): 897-909.

11. Norton PA. Pelvic floor disorders: the role of fascia and ligaments Clin Obstet Gynecol. 1993; 36(4): 926-38.

12. Symmonds RE, Williams TJ, Lee RA, Webb MJ. Posthysterectomy enterocele and vaginal vault prolapse. Am J Obstet Gynecol. 1981;140(8): 852-9.

13. Cervigni $M$, Natale $F$. The use of synthetics in the treatment of pelvic organ prolapse. Curr Opin Urol. 2001;11(4): 429-35.

14. Toozs-Hobson P, Boos K, Cardozo L. Management of vaginal vault prolapse. Br J Obstet Gynaecol. 1998;105(1): 13-7.

15. de Tayrac R, Picone O, Chauveaud-Lambling A, Fernandez H. A 2year anatomical and functional assessment of transvaginal rectocele repair using a polypropylene mesh. Int Urogynecol J Pelvic Floor Dysfunct. 2006; 17(2): 100-5. Epub 2005 May 21.

16. Jeon MJ, Jung HJ, Choi HJ, Kim SK, Bai SW. Is hysterectomy or the use of graft necessary for the reconstructive surgery for uterine prolapse? Int Urogynecol J Pelvic Floor Dysfunct. 2008; 19(3): 3515. Epub 2007 Oct 10.
17. Neuman M, Lavy $Y$. Conservation of the prolapsed uterus is a valid option: medium term results of a prospective comparative study with the posterior intravaginal slingoplasty operation. Int Urogynecol J Pelvic Floor Dysfunct. 2007; 18(8): 889-93. Epub 2006 Nov 30.

18. Bump RC, Mattiasson A, Bø K, Brubaker LP, DeLancey JO, Klarskov $P$, Shull BL, Smith AR. The standardization of terminology of female pelvic organ prolapse and pelvic floor dysfunction. Am J Obstet Gynecol. 1996;175(1): 10-7.

19. Lemos NL, Auge AP, Lunardelli JL, Carramão Sda S, Faria AL, Aoki T. Validation of the Pelvic Organ Prolapse Quantification Index (POP-Q-I): a novel interpretation of the POP-Q system for optimization of POP research. Int Urogynecol J Pelvic Floor Dysfunct. 2008;19(7): 995-7. Epub 2008 Jan 24.

20. Nesbitt RE Jr. Uterine preservation in the surgical management of genuine stress urinary incontinence associated with uterovaginal prolapse. Surg Gynecol Obstet. 1989;168(2): 143-7.

21. Weber AM, Richter HE. Pelvic organ prolapse. Obstet Gynecol. 2005;106(3): 615-34.

22. Maher C, Baessler K. Surgical management of anterior vaginal wall prolapse: an evidencebased literature review. Int Urogynecol J Pelvic Floor Dysfunct. 2006; 17(2): 195-201. Epub 2005 May 25.

23. Brubaker L. Fourth International Consultation On Incontinence (ICI) - Surgery For Pelvic Organ Prolapse Committee. Main Category: Urology / Nephrology Article Date: 26 Jul 2008 - 0:00 PDT Highlightswww.medicalnewstoday.com/articles/116186.php.

24. Deffieux X, Huel C, de Tayrac R, Bottero J, Porcher R, Gervaise A, Bonnet K, Frydman R, Fernandez H. [Vaginal mesh extrusion after transvaginal repair of cystocele using a prosthetic mesh: treatment and functional outcomes]. J Gynecol Obstet Biol Reprod (Paris). 2006; 35(7): 678-84.

25. DeLancey JO, Morley GW. Total colpocleisis for vaginal eversion. Am J Obstet Gynecol. 1997; 176(6): 1228-32; discussion 1232-5.

Recebido em 08/09/2008

Aceito para publicação em 13/11/2008

Conflito de interesse: nenhum

Fonte de financiamento: nenhuma

\section{Como citar este artigo:}

Carramão S, Auge APF, Pacetta AM, Ayrosa P, Duarte E, Lemos NL, Tsutomu. Estudo randômico da correção cirúrgica do prolapso uterino através de tela sintética de polipropileno tipo i comparando histerectomia versus preservação uterina. Rev Col Bras Cir. [periódico na Internet] 2009; 36(1). Disponível em URL: http://www.scielo.br/rcbc

\section{Endereço de correspondência:}

Silvia da Silva Carramão

E-mail: silviacarramao@superig.com.br 